PROCEEDINGS OF THE AMERICAN MATHEMATICAL SOCIETY

Volume 135, Number 5, May 2007, Pages 1241-1247

S $0002-9939(06) 08635-7$

Article electronically published on October 18, 2006

\title{
ON THE VARIETIES DEFINED BY RIEMANN-MUMFORD'S RELATIONS
}

\author{
RICCARDO SALVATI MANNI
}

(Communicated by Michael Stillman)

\begin{abstract}
In this paper we consider varieties defined by Riemann-Mumford's relations. An irreducible component of these varieties is related to Siegel modular varieties. We prove that in most cases Riemann-Mumford varieties are not irreducible.
\end{abstract}

\section{INTRODUCTION}

1.1. Let $\mathbb{H}_{g}$ be the Siegel upper half space, i.e., the set of complex symmetric matrices $\tau$ whose imaginary part is positive definite and let $S p(2 g, \mathbb{R})$ be the real symplectic group. $S p(2 g, \mathbb{R})$ acts transitively on $\mathbb{H}_{g}$ via

$$
\sigma \cdot \tau=(A \tau+B)(C \tau+D)^{-1}
$$

where $\sigma=\left(\begin{array}{cc}A & B \\ C & D\end{array}\right)$ is in $S p(2 g, \mathbb{R})$.

Let $\Gamma$ be a subgroup of finite index of the integral symplectic group and $k$ a half integer; thus a holomorphic function $f$ defined on $\mathbb{H}_{g}$ is called a modular form of weight $k$ and multiplier $\chi$ for $\Gamma$ if

$$
f(\sigma \cdot \tau)=f\left((A \tau+B)(C \tau+D)^{-1}\right)=\chi(\sigma) \operatorname{det}(C \tau+D)^{k} f(\tau)
$$

for all $\sigma \in \Gamma$. In the genus 1 case we require also the holomorphicity of $f$ at the cusps. We denote by $[\Gamma, k, \chi]$ the vector space spanned by such forms.

Letting $q$ denote an even positive integer and $m$ an element of $q^{-1} \mathbb{Z}^{g} / \mathbb{Z}^{g}$ for some $g \geq 1$, a Thetanullwert is defined by

$$
\theta\left[\begin{array}{c}
m \\
0
\end{array}\right](q \tau)=\sum_{p \in \mathbb{Z}^{g}} \exp \left(\pi i^{t}(p+m) q \tau(p+m)\right) .
$$

This is one of the simplest examples of modular forms of weight $\frac{1}{2}$ for $\Gamma_{g}(q, 2 q)$ and a suitable multiplier.

These Thetanullwerte induce well-defined maps

$$
\Theta_{g}(q): \Gamma_{g}(q, 2 q) \backslash \mathbb{H}_{g} \longrightarrow \mathbb{P}^{q^{g}-1}
$$

that are embeddings for all $g$ if $q \geq 4$ (cf. [6, 2]), and generically injective if $q=2$ (cf. [8]).

Received by the editors November 14, 2004 and, in revised form, November 20, 2005.

2000 Mathematics Subject Classification. Primary 11F46.

(C)2006 American Mathematical Society Reverts to public domain 28 years from publication 
1.2. Really, we know more; in fact, let $R_{g}(q)$ be the ring generated by such Thetanullwerte. It is a well-known fact that its integral closure $S_{g}(q)$ is the ring of modular forms $S(\Gamma(q, 2 q), \chi)$, with $\chi$ equal to the multiplier relative to the Thetanullwerte; cf. [2] and [6]. The map $\Theta_{g}(q)$ extends to the boundary of Satake's compactification of $\Gamma_{g}(q, 2 q) \backslash \mathbb{H}_{g}$, that is, $\operatorname{Proj}\left(S_{g}(q)\right)$.

In the ring $R_{g}(q)$ there are some standard relations. They are the quartic Riemann's relations and linear equations $X_{-m}=X_{m}$ with $m \in q^{-1} \mathbb{Z} / \mathbb{Z}$.

Let $\left(Q_{g}(q)\right)$ be the ring defined by the above equations. In this note we proceed to compare the associated projective varieties $\operatorname{Proj}\left(R_{g}(q)\right)$ and $\operatorname{Proj}\left(Q_{g}(q)\right)$. The final result of [7, page 202, states that $\Theta_{g}(q)\left(\operatorname{Proj}\left(R_{g}(q)\right)\right)$ is an irreducible component of $\operatorname{Proj}\left(Q_{g}(q)\right)$ when $q \geq 6$.

We shall show that, if $q \neq 2^{s}, \operatorname{Proj}\left(Q_{g}(q)\right)$ is not irreducible and hence it cannot be isomorphic to $\Theta_{g}(q)\left(\operatorname{Proj}\left(R_{g}(q)\right)\right)$.

In the last section, from a detailed analysis of $\operatorname{Proj}\left(Q_{1}(6)\right)$, we shall show how to reconstruct the ring of modular forms.

Finally, in this case we shall exhibit an explicit relation in the Thetanullwerte that is not a consequence of Riemann's relations.

\section{Riemann-Mumford's Relations}

2.1. We fix representatives for the characteristics. We choose the entries in the set

$$
\mathcal{F}(q)=\left[0, \frac{1}{q}, \ldots, \frac{q-1}{q}\right]
$$

and we set $1-m$ for the only characteristic $n$ such that $m+n \equiv 0 \bmod 1$.

In this section we shall consider the projective variety $\operatorname{Proj}\left(Q_{g}(q)\right)$ defined in $\mathbb{P}^{q^{g}-1}$ by the equations

$$
\begin{gathered}
X_{m}=X_{1-m}, \\
\left(\sum_{c \in \mathcal{F}(2)^{g}} \exp \left(4 \pi i^{t} c^{\prime} c\right) X_{a^{\prime}+d+c^{\prime}} X_{b^{\prime}+d+c}\right)\left(\sum_{c \in \mathcal{F}(2)^{g}} \exp \left(4 \pi i^{t} c^{\prime} c\right) X_{a+c} X_{b+c}\right) \\
=\left(\sum_{c \in \mathcal{F}(2)^{g}} \exp \left(4 \pi i^{t} c^{\prime} c\right) X_{a+d+c} X_{b+d+c}\right)\left(\sum_{c \in \mathcal{F}(2)^{g}} \exp \left(4 \pi i^{t} c^{\prime} c\right) X_{a^{\prime}+c} X_{b^{\prime}+c}\right)
\end{gathered}
$$

with $c^{\prime} \in \mathcal{F}(2)^{g}$ and $m, a, a^{\prime}, b, b^{\prime}, d \in \mathcal{F}(q)^{g}$ satisfying $a+b \equiv a^{\prime}+b^{\prime} \bmod 1$.

The link between these varieties and $\operatorname{Proj}\left(R_{g}(q)\right)$ is a consequence of the works of Mumford and Kempf; cf. [6] and [3]. In fact we have

Theorem 1. a) For all $q \geq 3, \Theta_{g}(q)$ is an immersion of $\Gamma_{g}(q, 2 q) \backslash \mathbb{H}_{g}$ in $\mathbb{P}^{q^{g}-1}$.

b) If $q$ is even and $q \geq 6$, then $\operatorname{Im}\left(\Theta_{g}(q)\right)$ is a Zariski open subset of $\operatorname{Proj}\left(Q_{g}(q)\right)$.

A proof of the above theorem can be found in [7]. Really in [7] the first statement is for $q \geq 4$.

We recall that in the case $q=3$ the injectivity of the map is proved in 44. Then we proved in [10] the injectivity on the tangent spaces. Moreover the case $q=2$ has been extensively studied in [8] and 9 . In [9] there are some inaccuracies, so at the moment we can say that the map $\Theta_{g}(2)$ is generically injective and it is injective when $g \leq 3$. Moreover we have to mention that when $q$ is even the maps $\Theta_{g}(q)$ extend to the boundary of the Satake compactification. 
2.2. According to the above facts, when $q$ is even, $\Theta_{g}(q)\left(\operatorname{Proj} S_{g}(q)\right)=\operatorname{Proj} R_{g}(q)$ is an irreducible reduced component of $\operatorname{Proj}\left(Q_{g}(q)\right)$.

Clearly we would like to show that equations (3) and (4) define $\left(\operatorname{Proj}_{g}(q)\right)$. Unluckily we will get a negative answer.

For example, from this labyrinth of polynomial relations, when $g=1$ and $q=6$, identifying $X_{1}$ with $X_{5}$ and $X_{2}$ with $X_{4}$, we obtain 2 relations, namely

$$
X_{0}^{2} X_{1} X_{3}+X_{0} X_{2} X_{3}^{2}=2 X_{1}^{2} X_{2}^{2}
$$

and

$$
X_{0}^{3} X_{2}+X_{1} X_{3}^{3}=X_{1}^{4}+X_{2}^{4} .
$$

(We multiplied the indices by 6 to avoid heavier notation.)

The projective line of equations $X_{1}=X_{2}=0$ is contained in $\operatorname{Proj} Q_{6}$, which is not irreducible.

We shall prove that this is a general fact, when $q \equiv 2 \bmod 4$. In fact, if we set $X_{a}=0$ when $a \notin \mathcal{F}(2)^{g}$, the equations become trivial unless $\left\{a^{\prime}+d, b^{\prime}+d, a, b\right\}$ or $\left\{a^{\prime}, b^{\prime}, a+d, b+d\right\}$ are in $\mathcal{F}(2)^{g}$.

Each of these configurations implies that $2 d \in \mathcal{F}(2)^{g}$, and, in these cases, we get $d \in \mathcal{F}(2)^{g}$.

We remark that these are exactly the quartic relations among Thetanullwerte with half integral characteristics and, it is a well-known fact, that these relations do not exist.

To be clearer, it can be easily verified that equation (4) becomes

$$
\begin{aligned}
& \left(\sum_{c \in \mathcal{F}(2)^{g}} \exp \left(4 \pi i^{t} c^{\prime} c\right) X_{a^{\prime}+c^{\prime}} X_{b^{\prime}+c}\right)\left(\sum_{c \in \mathcal{F}(2)^{g}} \exp \left(4 \pi i^{t} c^{\prime} c\right) X_{a+c} X_{b+c}\right) \\
& =\left(\sum_{c \in \mathcal{F}(2)^{g}} \exp \left(4 \pi i^{t} c^{\prime} c\right) X_{a+c} X_{b+c}\right)\left(\sum_{c \in \mathcal{F}(2)^{g}} \exp \left(4 \pi i^{t} c^{\prime} c\right) X_{a^{\prime}+c} X_{b^{\prime}+c}\right) .
\end{aligned}
$$

These are obviously tautological, so we proved the following.

Theorem 2. Let us assume $q \equiv 2 \bmod 4$. Then for any $g$ the projective variety $\operatorname{Proj}\left(Q_{g}(q)\right)$ defined by the equations (3) and (4) has more than an irreducible component. In particular it contains a linear variety of dimension at least $2^{g}-1$.

Really, with some modifications we can prove more; in fact we have

Theorem 3. Let us assume $q \neq 2^{s}$ for some positive integer $s>1$. Then for any $g$ the projective variety $\operatorname{Proj}\left(Q_{g}(q)\right)$ defined by the equations (3) and (44) has more than an irreducible component.

Proof. Let us assume $q=2^{s} o$, with $o$ an odd number bigger than 1 and $\operatorname{Proj}\left(Q_{g}(q)\right)$ irreducible.

If we set $X_{a}=0$ when $a \notin \mathcal{F}\left(2^{s}\right)^{g}$, also in these cases the equations (4) become trivial unless $\left\{a^{\prime}+d, b^{\prime}+d, a, b\right\}$ or $\left\{a^{\prime}, b^{\prime}, a+d, b+d\right\}$ are in $\mathcal{F}\left(2^{s}\right)^{g}$.

Each of these configurations implies that $2 d \in \mathcal{F}\left(2^{s}\right)^{g}$, and we get $d \in \mathcal{F}\left(2^{s}\right)^{g}$. These are exactly the equations defining $\operatorname{Proj}\left(Q_{g}\left(2^{s}\right)\right)$.

Thus, if $L_{q}$ is the linear space defined by $X_{a}=0$ when $a \notin \mathcal{F}\left(2^{s}\right)^{g}$, we have

$$
L_{q} \cap \operatorname{Proj}\left(Q_{g}(q)\right)=\operatorname{Proj}\left(Q_{g}\left(2^{s}\right)\right) .
$$

Now if $\operatorname{Proj}\left(Q_{g}(q)\right)$ is irreducible we know that its dimension is exactly $(1 / 2) g(g+1)$ and in any case $\operatorname{Proj}\left(Q_{g}\left(2^{s}\right)\right)$ has dimension greater than or equal 
to $(1 / 2) g(g+1)$, since $\operatorname{Proj} R_{g}(q)$ is an irreducible component. Thus we get a contradiction once we prove that the inclusion

$$
L_{q} \cap \operatorname{Proj}\left(Q_{g}(q)\right) \subset \operatorname{Proj}\left(Q_{g}(q)\right)
$$

is proper.

Letting $\tau \in \mathbb{H}_{g}$ be purely imaginary, i.e. $\tau=i y$, we verify that $\Theta_{g}(q)(i y)$ in $\mathbb{P}^{q^{g}-1}$ has all entries different from 0 . This is an immediate consequence of the definition of Thetanullwerte, since

$$
\theta\left[\begin{array}{c}
m \\
0
\end{array}\right](i q y)=\sum_{p \in \mathbb{Z}^{g}} \exp (-\pi q y[p+m])
$$

is the convergent sum of positive terms.

This shows that

$$
\Theta_{g}(q)(i y) \notin L_{q} .
$$

These results are useful for a better understanding of the variety $\operatorname{Proj} S_{g}(q)$ at least for small values of $g$. This will be discussed in the next section.

\section{An example}

3.1. Using the results of the last section we will obtain a good description of $\operatorname{Proj} S_{1}(6)$. We know that it has exactly 24 cusps. It is a Riemann surface of genus 13 , since it is a Galois covering of degree 12 of $\operatorname{Proj} S_{1}(2) \cong \mathbb{P}^{1}$ and ramifies only on the 6 cusps of $\operatorname{Proj} S_{1}(2)$.

Let $Y_{1}$ and $Y_{2}$ be the quartics defined by (15) and (6); thus $\operatorname{Proj} Q_{1}(6)=Y_{1} \cap Y_{2}$ contains 4 lines $L_{1}, L_{2}, L_{3}, L_{4}$ of equations

$$
\begin{gathered}
X_{1}=0, X_{2}=0 ; \quad X_{2}-X_{0}=0, X_{3}-X_{1}=0 ; \\
X_{2}-\phi^{4} X_{0}=0, X_{3}-\phi^{8} X_{1}=0 ; \quad X_{2}-\phi^{8} X_{0}=0, X_{3}-\phi^{4} X_{1}=0
\end{gathered}
$$

with $\phi=\exp \left(\frac{2 \pi i}{12}\right)$. Thus we can write

$$
\operatorname{Proj} Q_{1}(6)=L_{1} \cup L_{2} \cup L_{3} \cup L_{4} \cup \mathcal{C} .
$$

It has exactly 24 singular points. They are

$$
\begin{gathered}
{[1,0,0,1],[1,0,0,-1],[1,0,0, i],[1,0,0,-i],[0,0,0,1],[1,0,0,0],} \\
{[1,0,1,0],[1, i, 1, i],[1,-i, 1,-i],[1,1,1,1],[1,-1,1,-1],[0,1,0,1],} \\
{\left[1,0, \phi^{4}, 0\right],\left[1, \phi, \phi^{4},-i\right],\left[1, \phi^{7}, \phi^{4}, i\right],\left[1, \phi^{-2}, \phi^{4},-1\right],\left[1, \phi^{4}, \phi^{4}, 1\right],\left[0, \phi^{4}, 0,1\right],} \\
{\left[1,0, \phi^{8}, 0\right],\left[1, \phi^{-1}, \phi^{8},-i\right],\left[1, \phi^{5}, \phi^{8}, i\right],\left[1, \phi^{2}, \phi^{8},-1\right],\left[1, \phi^{8}, \phi^{8}, 1\right],\left[0, \phi^{8}, 0,1\right] .}
\end{gathered}
$$

The first set of six points is contained in $L_{1}$; the second, the third and the fourth set in $L_{2}, L_{3}, L_{4}$ respectively.

Since $\Theta_{g}(q)$ is $\Gamma_{g} / \Gamma_{g}(q, 2 q)$-equivariant and it can be easily verified that the above points have nontrivial stabilizer for the action of $\Gamma_{1}(2,4) / \Gamma_{1}(6,12)$, they are the image of the cusps and have the same singularity.

Hence to prove that $\operatorname{Proj} S_{1}(6) \cong \operatorname{Proj} R_{1}(6)$ it is enough to check that a singularity is nodal.

This can be easily verified at the point $[1,0,0,0]$. In fact, passing to affine coordinates, we have the following equations:

$$
x z+y z^{2}=2 x^{2} y^{2}
$$


and

$$
y+x z^{3}=x^{4}+y^{4} .
$$

Then obtaining $y$ in the second equation and substituting in the first we get that the principal tangent has equation $x z=0$.

Thus $\operatorname{Proj}_{1}(6) \cong \operatorname{Proj} R_{1}(6)$ is a curve in $\mathbb{P}^{3}$ whose automorphism group has order divisible by

$$
\left|\Gamma_{1} / \pm \Gamma_{1}(6,12)\right|=288 .
$$

3.2. A priori we cannot say that $\operatorname{Proj} S_{1}(6) \cong \mathcal{C}$; in fact we have not shown that $\mathcal{C}$ is irreducible.

Since $\mathcal{C}$ is smooth, it is enough to show that it is connected.

We observe that the quartic $Y_{2}$ is smooth and the effective divisor $\mathcal{C}$ induces a linear system on the quartic that is equivalent to

$$
\left(4 H-L_{1}-L_{2}-L_{3}-L_{4}\right) \text {. }
$$

Here with $H$ we denote the hyperplane section of $\mathbb{P}^{3}$ restricted to $Y_{2}$.

Let us consider the linear system $H-L_{i}, i=1, \ldots, 4$. These induce maps $f_{i}: Y_{2} \rightarrow \mathbb{P}^{1}$ that describe one-dimensional families of cubic curves in the quartic surface.

Each of these systems is without base points ; in fact this can be easily proved for points of the quartic that are not on the line and for the points on the line we remark that if one is a base point, then it should be a singular point of $Y_{2}$, but this is impossible.

Consequently the linear system $\left(4 H-L_{1}-L_{2}-L_{3}-L_{4}\right)$ is without base points and thus it induces a morphism

$$
f: Y_{2} \rightarrow \mathbb{P}^{n} .
$$

Moreover we have that $\operatorname{dim}\left(f\left(Y_{2}\right)\right)=2$; in fact for each of the maps $f_{i}$ the generic fiber is a cubic and the generic fiber of the map

$$
f_{1} \times f_{2}: Y_{2} \rightarrow \mathbb{P}^{1} \times \mathbb{P}^{1}
$$

is finite, since it is the intersection of two cubics contained in the quartic surface that are not in the same plane.

As a consequence of Zariski's Main Theorem (cf. 1], p.280, Ex.11.3), we get that all divisors in $\left|4 H-L_{1}-L_{2}-L_{3}-L_{4}\right|$, and in particular $\mathcal{C}$, are connected. Hence we get

$$
\operatorname{Proj} S_{1}(6) \cong \mathcal{C} .
$$

We are grateful to Marco Manetti for suggesting this proof.

Really one could prove more; in fact with some computation it is possible to show that the divisor $\left(4 H-L_{1}-L_{2}-L_{3}-L_{4}\right)$ satisfies the condition of a criterion (Nakai-Moishezon) of ampleness.

3.3. Now we shall treat the relations in $R_{1}(6)$; in particular we look for relations that are not induced by Riemann's relations.

For this reason we analyze the graded rings $Q_{1}(6)$ and $S_{1}(6)$.

For the first graded ring we have that its Poincaré series is

$$
P(t)=\sum_{k=0}^{\infty} \operatorname{dim} Q_{1}(6)_{k} t^{k}=\frac{\left(1-t^{4}\right)^{2}}{(1-t)^{4}} .
$$


Thus we have

$$
\operatorname{dim} Q_{1}(6)_{4}=33, \quad \operatorname{dim} Q_{1}(6)_{6}=65 .
$$

Moreover from [5], p. 61, we get

$$
P^{\prime}(s)=\sum_{k=0}^{\infty} \operatorname{dim} S_{1}(6)_{2 k} s^{k}=\frac{1+10 s+13 s^{2}}{(1-s)^{2}}
$$

Consequently we get

$$
\operatorname{dim} S_{1}(6)_{4}=\operatorname{dim}\left[\Gamma_{1}(6,12), 2, i d\right]=36, \operatorname{dim} S_{1}(6)_{6}=\operatorname{dim}\left[\Gamma_{1}(6,12), 3, i d\right]=60 .
$$

Hence we have that the Thetanullwerte satisfy some relations in degree 6 , in fact, using the decomposition of these spaces with respect to some characters of $\Gamma_{1}(2,4)$.

Theorem 4. The following relation holds:

$$
\begin{aligned}
& 2\left(\theta\left[\begin{array}{l}
1 \\
0
\end{array}\right]^{4}(6 \tau)-\theta\left[\begin{array}{l}
2 \\
0
\end{array}\right]^{4}(6 \tau)\right) \theta\left[\begin{array}{l}
1 \\
0
\end{array}\right](6 \tau) \theta\left[\begin{array}{l}
2 \\
0
\end{array}\right](6 \tau) \\
& -\left(\theta\left[\begin{array}{l}
0 \\
0
\end{array}\right]^{4}(6 \tau)-\theta\left[\begin{array}{l}
3 \\
0
\end{array}\right]^{4}(6 \tau)\right) \theta\left[\begin{array}{l}
0 \\
0
\end{array}\right](6 \tau) \theta\left[\begin{array}{l}
3 \\
0
\end{array}\right](6 \tau) .
\end{aligned}
$$

This relation is not induced from Riemann's relations.

Proof. To avoid the problem induced by the multiplier we shall consider the modular form

$$
\begin{aligned}
g(\tau)= & 2\left(\theta\left[\begin{array}{l}
1 \\
0
\end{array}\right]^{4}-\theta\left[\begin{array}{l}
2 \\
0
\end{array}\right]^{4}\right) \theta\left[\begin{array}{l}
1 \\
0
\end{array}\right]^{2} \theta\left[\begin{array}{l}
2 \\
0
\end{array}\right]^{2} \\
& \left.-\left(\theta\left[\begin{array}{l}
0 \\
0
\end{array}\right]^{4}-\theta\left[\begin{array}{l}
3 \\
0
\end{array}\right]^{4}\right) \theta\left[\begin{array}{l}
0 \\
0
\end{array}\right]\right) \theta\left[\begin{array}{l}
3 \\
0
\end{array}\right] \theta\left[\begin{array}{l}
1 \\
0
\end{array}\right] \theta\left[\begin{array}{l}
2 \\
0
\end{array}\right],
\end{aligned}
$$

where $g(\tau)$ is a cusp form, since it vanishes at all 24 cusps of $\operatorname{Proj} S_{1}(6)$. An easy, but rather tedious computation, involving the Fourier coefficients of $g(\tau)$, shows that its vanishing at the cusps is so high that $g(\tau) \equiv 0$.

Elementary computations show that the projective lines $L_{1}, L_{2}, L_{3}, L_{4}$ are not contained in the surface $Y_{3}$ of degree 6 defined by equation (14); consequently the above relation is not induced from Riemann's relations.

We remark that the curve $Y_{1} \cap Y_{2} \cap Y_{3}$ is isomorphic to $\operatorname{Proj}\left(S_{1}(6)\right)$.

However we have numerical evidence that we did not give a complete description of all relations among the Thetanullwerte; however we can find all other relations using the action of $\Gamma_{1} / \Gamma_{1}(6,12)$.

\section{REFERENCES}

[1] Hartshorne,R.: Algebraic geometry. Graduate Texts in Mathematics, 52 xvi+496 pp., Springer-Verlag, New York-Heidelberg, 1977. MR0463157 (57:3116)

[2] Igusa, J.: Theta Functions. Grundlehren der mathematischen Wissenschaften, 194. BerlinHeidelberg-New York: Springer (1972). MR0325625 (48:3972)

[3] Kempf, G.: Linear systems on abelian varieties. Amer. J. Math. 111 , 65-94 (1989). MR0980300 (90d:14046)

[4] Lange, H., Birkenhake, C.: Complex Abelian Varieties. Grundlehren der mathematischen Wissenschaften, 302. Berlin-Heidelberg-New York: Springer (1992). MR.1217487 (94j:14001) 
[5] Miyake, T.: Modular forms. Translated from the Japanese by Yoshitaka Maeda, $\mathrm{x}+335 \mathrm{pp}$. Springer-Verlag, Berlin, (1989). MR.1021004 (90m:11062)

[6] Mumford, D.: On the Equations Defining Abelian Varieties I, II, III. Invent. Math. 1 287-354 (1966), 3 71-135, 215-244 (1967). MR0204427(34:4269) MR0219541(36:2621) MR0219542 $(36: 2622)$

[7] Mumford, D.: Tata Lectures on Theta III. Progress in Mathematics, 97. Boston-Basel-Berlin: Birkhäuser (1991). MR1116553 (93d:14065)

[8] Salvati Manni, R.: On the Projective Varieties Associated with some Subrings of the Ring of Thetanullwerte. Nagoya Math. J. 133 , 71-83 (1994). MR.1266363 (95f:14088)

[9] Salvati Manni, R.: Modular varieties with level 2 theta structure. Amer. J. Math. 111 , 1489-1511 (1994). MR1305875 (96c:11051)

[10] Salvati Manni, R.: On the differential of applications defined on the moduli spaces of p.p.a.v. with level theta structure. Math.Z. 221 , 231-241 (1996). MR.1376295 (96k:11059)

Dipartimento di Matematica, Università di Roma la Sapienza, Piazzale Aldo Moro 2, I-00185 Roma, Italy

E-mail address: salvati@mat.uniroma1.it 